CLAWAR 2018: 21st International Conference on Climbing and

Walking Robots and the Support Technologies for Mobile Machines,

Panama City, Panama, 10-12 September 2018

\title{
DESIGN OF A GRIPPING MOVEMENT CONTROL SYSTEM FOR A ROBOTIC HAND PROSTHESIS
}

\author{
RUTTY CEDEÑO ARANA ${ }^{1}$, LISBETH MENA ${ }^{1}$, HECTOR MONTES ${ }^{2,3}$, RICARDO $^{2}$ \\ CAJO ${ }^{1}$ \\ ${ }^{1}$ ESPOL Polytechnic University, Escuela Superior Politécnica del Litoral, ESPOL. \\ (Facultad de Ingeniería en Electricidad y Computación), Campus Gustavo Galindo Km. \\ 30.5 Via Perimetral, P.O. Box 09-01-5863, Guayaquil, Ecuador. \\ ${ }^{2}$ Field and Service Robotics Group, Centre for Automation and Robotics-CAR (CSIC- \\ UPM), Spain. \\ ${ }^{3}$ Faculty of Electrical Engineering, Universidad Tecnológica de Panamá, Panama. \\ rutacede@espol.edu.ec,lismena@espol.edu.ec,hector.montes@csic.es, \\ rcajo@espol.edu.ec
}

\begin{abstract}
In this paper, a description of a control system for basic grip movements for a low-cost hand prosthesis is presented. The idea is to provide movements to those people who do not have fingers due to congenital malformations, considering that the movements of their wrists are complete. The wrist movements are acquired by means of the Myo bracelet, which has wireless communication. The Myo bracelet has eight myoelectric sensors and an IMU, i.e., non-invasive sensors that are positioned around the forearm. The gestures read by means of the Myo bracelet are processed by the control system to send the commands to the small electric actuators installed in the prototype hand prosthesis.
\end{abstract}

Keywords: Robotic hand prosthesis; gripping movements; control system; non-invasive sensors; Myo bracelet.

\section{Introduction}

There are several causes why a person can have a physical disability, ranging from congenital malformations to degenerative diseases or accidents. The loss or absence of upper limbs of the human body is a limiting factor for the development of activities of daily life. The hand is one of the most useful parts of the body and the most used in daily activities, so the loss of it or lack of fingers is a drawback that greatly diminishes the functionality of the body. Some of the contributions that help improve the quality of life in people who have this condition, are robotic hand prostheses [1], [2].

The hand prosthesis that is proposed in this work will be controlled using myoelectric signals acquired from the forearm muscles and the use of IMU data (inertial measurement unit). In this work, the device used is the Myo bracelet, 
developed by Thalmic Labs, which has an inertial measurement unit of nine axes and EMG (electromyography) muscle activity sensors. This bracelet is placed on the upper part of the forearm. In addition, it has a wireless communication interface, with low power consumption Bluetooth 4.0, which provides access to the raw data corresponding to IMU and EMG sensors [3]. The acquired data will be sent to a nano Arduino controller card in which the libraries and the corresponding program will be loaded to carry out an individual control of five servomotors in charge of the movements of flexion and extension of each finger of the prosthesis.

This project emerged with the motivation to develop a low-cost prosthesis to allow carry out gripping basic movements for Ecuador citizens that have this kind of problem. However, it has been considered a specific case of a person who has a birth defect in one hand. It has been diagnosed as metacarpal agenesis, which refers to the absence of partial or total of an organ or a tissue of the organism. In this case, the absence of fingers. However, the movements of the wrist are complete [4].

\section{System Description}

\subsection{Description of the elements of the prototype}

The Myo bracelet used in this project has its own processor called ARM Cortex M4Processor [5], to process the data of the 8 EMG sensors arranged as a bracelet, which thanks to its expandable band adapt to the shape of the forearm. It also has a gyroscope, an accelerometer and a magnetometer.

The standard communication and data acquisition of the Myo bracelet is realized by connecting it to a computer, through its USB Bluetooth adapter. This concept avoids the possibility of developing a portable prototype and the direct connection with other electronic platforms. Therefore, it is necessary to design and develop another route of data transmission. Consequently, for this project, an integrated Bluetooth 4.0 device with low power consumption has been employed [6].

The general structure of the software is composed by means of two libraries called MyoBridge and MyoIMUGestureController. The first library allows bidirectional communication with the Myo bracelet, and the second is responsible for reading and recognizing gestures. Besides, the second library contains the main program, where the control parameters are established and conditions for the generation of movements of the prosthesis. 


\subsection{Control system architecture}

The control of the hand prosthesis shown in Figure 1 is based on the acquisition of IMU and EMG data captured by the Myo bracelet, which is positioned on the upper part of the forearm. It communicates wirelessly to a nano-Arduino controller card. This controller uses a Bluetooth module with low power consumption as an intermediary. The controller card is responsible for processing the signals captured by the Myo bracelet and transfers them into patterns of grip movements for a 3D printed prosthesis [7], by activating the servomotors.

The control for grip movements for the hand-held robotic prosthesis described in this work is based on the programming of an Arduino Nano board. This decision has been taken with the objective to develop a low-cost usable prototype and the simple programming language that provides the Arduino system. However, the knowledge that will be acquired in this work can be extrapolated using other types of electronic devices.

In addition, it is also considered the suitable motions of the prosthesis for the user comfort. The Myo bracelet has pre-established gesture recognition, movement tracking and measurement of muscle activity. As has been mentioned above, this system uses a Bluetooth 4.0 module in order to transmit and receive data in wireless mode. Because most low-power Bluetooth models have a very limited feature set, none of these is capable, under its factory state, of directly connecting to the Myo bracelet. This problem has been solved by loading a custom firmware MyoBridge, with CCLoader program and making the respective hardware connections.

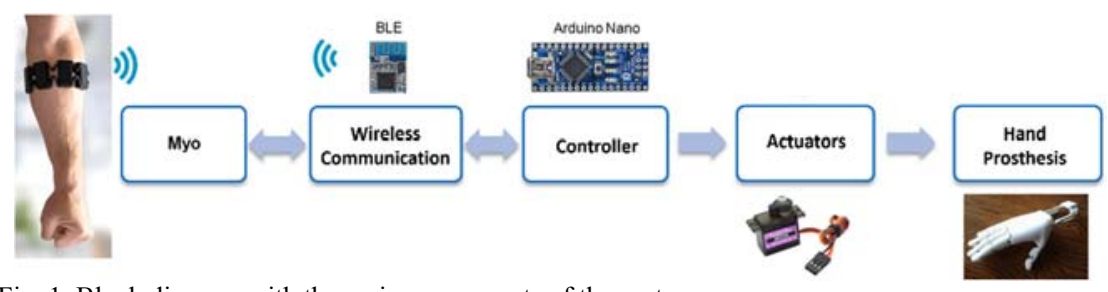

Fig. 1. Block diagram with the main components of the system.

\section{Results}

\subsection{Reading status}

The prosthesis has three LED indicators, a red-LED to show low battery level, an RGB-LED shows the gesture and a blue-LED shows reading state.

The state of data reading begins with a signal of muscular activity or a movement that produce force signals. The reading period it is programmed to be 
active for a lapse of 6 seconds, approximately. It may end or be blocked by the same muscle activity signal that was initially performed. For visualization, a blue LED is used, which will remain on only in the time of data acquisition.

\subsection{Gesture recognition}

Although the Arduino serial monitor is a useful tool for visualizing the gesture interpreted by the program, it only can use during the simulations. Therefore, another way of knowing the gesture that was recognized or not recognized is through the RGB-LED indicator, as shown in Figure 2.

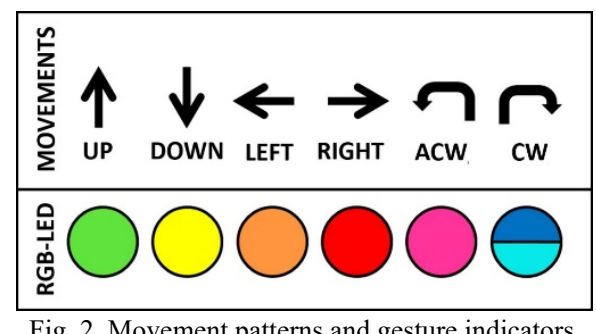

\subsection{Movements of the prosthesis}

Currently, the prosthesis movements are established in response to the registered gestures. In this case, it has been registered four movements of signs and five movements of grip, as shown in Table 1.

\begin{tabular}{cl}
\multicolumn{2}{c}{ Table 1. List of movements of the prosthesis } \\
\hline Movement & Description \\
\hline Grip 1 & Hold hands \\
Grip 2 & Hold bottle \\
Grip 3 & Hold a cup \\
Grip 4 & Hold mouse (Principal and secondary buttons) \\
Grip 5 & Hold with index and middle fingers \\
Sign 1 & Number 1, Point with the index finger \\
Sign 2 & Number 2, Extension only the index and the middle fingers \\
Sign 3 & Number 3, Flexion of the thumb and the index finger \\
Sign 4 & Open hand, extension of the 5 fingers \\
\hline
\end{tabular}

Next, each one of the established grip movements will be described:

- Hold hands: This position is shown in Figure 3(a). It is achieved when the little, middle and index fingers are contracted, in order to be able to give greetings with a handshake. 
- Hold bottle: This position is shown in Figure 3(b) and is achieved when all fingers except the thumb are contracted, in order to hold cylindrical objects.

- Holding mouse: This position is shown in Figure 3(c) and is achieved by contracting the little finger, ring finger, and thumb. These fingers in conjunction with the support of the palm control the sliding of a mouse on a surface flat. In addition, once the person is in this position, two more movements are unlocked, i.e., right click with the middle finger and left click with the index finger. This grip movement pretends to convert the prosthesis into a tool.

- Hold cup: This position is shown in Figure 3(d) and is achieved by contracting the little and ring fingers, so that the middle and index fingers are placed in the ear of a cup, then they contract and hold.

- Hold with index and middle fingers: This position is shown in Figure 3(e) and is achieved when the index and middle finger are closed to hold small objects such as tennis balls, keychains, etc.

The Figure 3(f) shows the prosthesis prototype used by the person with congenital malformation.

For gripping movements, a force resistive sensor (FSR) was installed in the fingerprint position of the index finger. Its functionality is to allow feedback control loop when the grip has been carried out. As a future work, FSR will be installed on all fingers to have new control conditions.

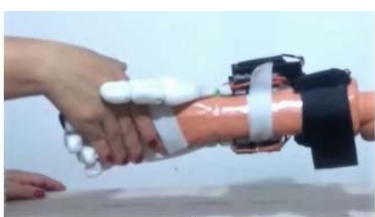

a)

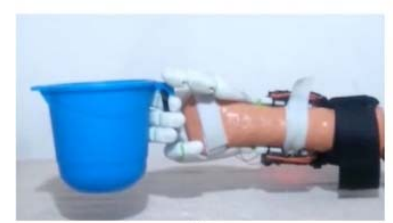

d)

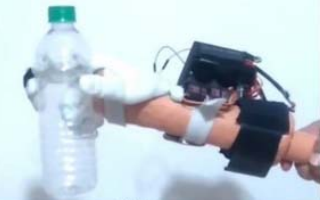

b)

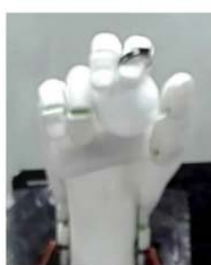

e)

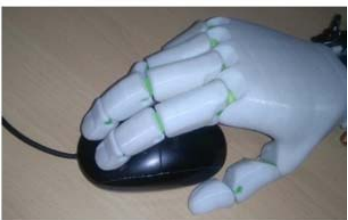

c)

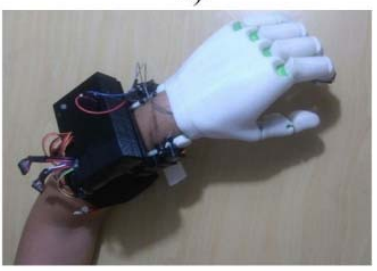

f)

Fig. 3. Movement patterns and gesture indicators. 


\section{Conclusions}

The control of the hand prosthesis was made by means of Bluetooth 4.0 communication between the Myo Bracelet and the Arduino Nano card, with this the use of cables for the capture of the signals coming from the Myo bracelet sensors was eliminated. The reading patterns that the Myo bracelet should read are generated from movements of the forearm that are achieved with complete naturalness, which represents a great advantage since it facilitates the handling of the prosthesis.

The use of 3D printing technology can easily the manufacture of custom prostheses, since most people do not have the same deformation patterns in their extremities. In this work, a custom prosthesis has been designed and implemented to be used by the person that collaborates with the project.

The current prototype can perform nine movements, of which four are of sign making and five are grip movements. While the printed prosthesis allowed to validate the grip movements defined in the control system, this presented any drawbacks due to the material used in its printing. The rigid plastic generated slippages in most of the textures during the experimental tests using the prosthesis.

For future works, it will be acquired servomotors with torques higher than the current servomotors in order to exert sufficient force on the tendons of the hand to move and lift objects with considerable weight. Additionally, the 3D printed hand will be cover with special material in order to avoid the slippages.

\section{Acknowledgements}

Rutty Cedeño, Lisbeth Mena and Ricardo Cajo thank the support of the ESPOL Polytechnic University, Ecuador. Hector Montes thanks the National System of Researchers of Panama (SNI-SENACYT) by the support to their members. Hector Montes also acknowledges support from the Centre for Automation and Robotics CSIC-UPM and Universidad Tecnológica de Panamá.

\section{References}

1. J. Zuniga, D. Katsavelis, J. Peck, J. Stollberg, M. Petrykowski, A. Carson, and C. Fernandez. Cyborg beast: a low-cost 3d-printed prosthetic hand for children with upper-limb differences. BMC Res Notes 8, No. 1, p. 10. (2015)

2. M. D. R. Ahsan, M. I. Ibrahimy, and O. O. Khalifa. Advances in electromyogram signal classification to improve the quality of life for the disabled and aged people. Journal of Computer Science 6(7), 706. (2010) 
3. Thalmic Labs, (Nov. 2015). Meet the Man With a Myo-Controlled Robotic Arm. [online] The Lab. Available in: http://developerblog.myo.com/meetthe-man- with-a-myo-controlled-robotic-arm/

4. M.A. Tonkin, S. K. Tolerton, T. J. Quick, I. Harvey, R. D. Lawson, N.C. Smith, and K. C. Oberg. Classification of congenital anomalies of the hand and upper limb: development and assessment of a new system. Journal of Hand Surgery 38(9), 1845-1853, (2013).

5. Myo Gesture Control Armband, Myo.com, (2014), [Online]. Available in: https://www.myo.com/techspecs.

6. JNHuaMao Technology Company. Bluetooth 4.0 BLE module, Datasheet. (2014).

7. Flexy-Hand2. Thingiverse.com, (2014), [Online]. Available in: https://www.thingiverse.com/thing:380665.

8. C. Setiawan. Tangan Robot Bionik dengan Kendali Otot Berbasis EMG. Journal Tahun, (2015).

9. F. V. Tenore, A. Ramos, A. Fahmy, S. Acharya, R. Etienne-Cummings and N. V. Thakor. Decoding of Individuated Finger Movements Using Surface Electromyography. IEEE Trans Biomed Eng. 56(5), 1427, (2009). 